\title{
Modeling and Eigenfrequency Analysis of Sound-Structure Interaction in a Rectangular Enclosure with Finite Element Method
}

\author{
Samira Mohamady, ${ }^{1}$ Raja Kamil Raja Ahmad, ${ }^{1}$ Allahyar Montazeri, ${ }^{2}$ Rizal Zahari, ${ }^{3}$ \\ and Nawal Aswan Abdul Jalil ${ }^{4}$ \\ ${ }^{1}$ Departement of Electrical and Electronic Engineering, Universiti Putra Malaysia, 43400 UPM SERDANG, Selangor, Malaysia \\ ${ }^{2}$ Faculty of Electrical Engineering, Iran University of Science and Technology, Narmak 16846-13114, Tehran, Iran \\ ${ }^{3}$ Department of Aerospace Engineering, Universiti Putra Malaysia, 43400 UPM SERDANG, Selangor, Malaysia \\ ${ }^{4}$ Departement of Mechanical and Manufacturing Engineering, Universiti Putra Malaysia, 43400 UPM SERDANG, Selangor, Malaysia
}

Correspondence should be addressed to Samira Mohamady, arimasim@ieee.org

Received 26 July 2009; Accepted 20 November 2009

Recommended by Massimo Viscardi

\begin{abstract}
Vibration of structures due to external sound is one of the main causes of interior noise in cavities like automobile, aircraft, and rotorcraft, which disturb the comfort of passengers. Accurate modelling of such phenomena is required in eigenfrequency analysis and in designing an active noise control system to reduce the interior noise. In this paper, the effect of periodic noise travelling into a rectangular enclosure is investigated with finite element method (FEM) using COMSOL Multiphysics software. The periodic acoustic wave is generated by a point source outside the enclosure and propagated through the enclosure wall and excites an aluminium flexible panel clamped onto the enclosure. The behaviour of the transmission of sound into the cavity is investigated by computing the modal characteristics and the natural frequencies of the cavity. The simulation results are compared with previous analytical and experimental works for validation and an acceptable match between them were obtained.
\end{abstract}

Copyright ( $) 2009$ Samira Mohamady et al. This is an open access article distributed under the Creative Commons Attribution License, which permits unrestricted use, distribution, and reproduction in any medium, provided the original work is properly cited.

\section{Introduction}

Modelling of sound propagation in an enclosure is of considerable importance in the design and analysis of an active noise control system. Reduction of noise in aircrafts, automobiles and house appliances is important due to their annoying effects on human. Many of these applications can be modelled by a 3D cavity with a flexible boundary condition on one of its sides. An approach to this modelling is to consider an enclosure with rigid boundary conditions $[1,2]$ and extends to the case where one of its boundaries is considered as flexible [3, 4]. Excitation of the flexible plate by sound source will cause vibrations on the plate and induces noise inside the cavity. Due to the coupling between structural vibrations and acoustical field, these systems are termed vibro-acoustic systems. Several analytical and experimental studies have been conducted to study the behaviour of these vibro-acoustic systems. In the earliest studies in [5-7] a comprehensive modal based theoretical framework of sound field inside an enclosure has been developed. Asymptotic modal analysis technique has been proposed [8] to analyze such problems and has been shown to have advantages over traditional methods used for solving dynamic problems with a large number of modes. Furthermore, a mechanics-based analytical model has also been developed [9] to address the interactions between a panel and the sound field inside a rectangular enclosure. In this work, a rectangular enclosure with a flexible panel with piezoelectric actuators attached to it $\mathrm{w}$ modelled. The studies in [10] for an irregular enclosure with two flexible panels are extended here. Finite element models have been constructed to study similar problems $[11,12]$. In several studies, the geometry of the enclosure was considered to be non rectangular, but the same modal analysis strategy was used to study the behaviour of the sound travelling within it [13].

In modelling the effect of coupling between the flexible plate and the enclosure, both simply supported and clamped boundaries have been used, but several studies used only 


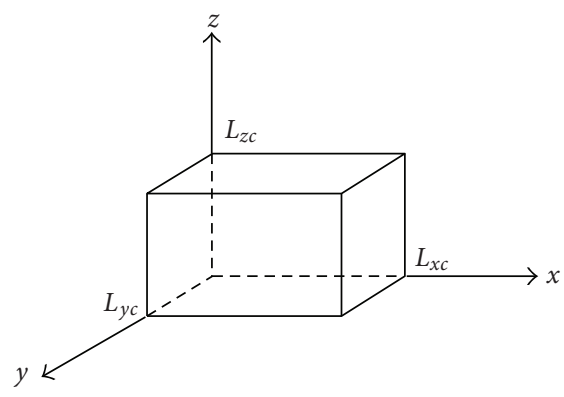

(a)

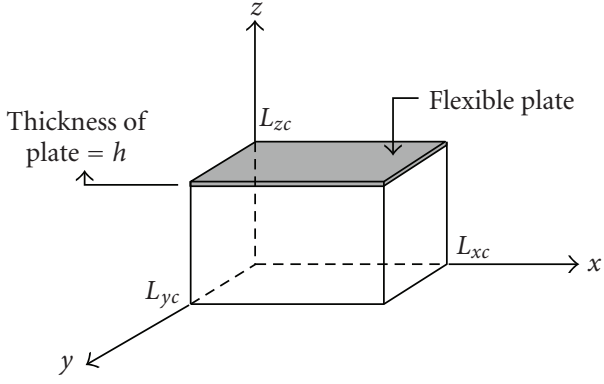

(b)

Figure 1: Dimension of a rectangular enclosure.

simply supported $[4,14,15]$ because the analytical derivation of the model for the coupled system is less complex. Therefore, finite element technique is more useful in modelling clamped boundaries.

Computational techniques have been employed to solve the vibro-acoustic problems thanks to the rapid advancement of computing power. Finite element and boundary element methods are two examples of computational techniques, which can be used to study the characteristics of sound radiation from a box-type structure. The boundary element method provides a versatile means of solving acoustic radiation problem in arbitrary shaped regions, but in order to be used efficiently the elements must be smaller than a fraction of the acoustic wavelength. Therefore, in problems with three-dimensional geometry, modelling can be performed on a desktop computer just for frequencies up to a few tens or at most hundreds of $\mathrm{Hz}$ [16].

In this paper a reliable finite element model for the analysis of the vibro-acoustic behaviour of a rectangular enclosure is developed. It is assumed that the sound is transmitted through the flexible panel that is attached to the enclosure with clamped boundary conditions. The modal analysis of such an enclosure was simulated and the results are compared with the analytical and experimental results obtained in related study [3]. An error analysis of the obtained resonant frequencies was used to validate the developed model. The finite element modelling was performed using COMSOL Multiphysics software that provides exclusive structural and acoustical modules as well as the ability of connecting them together to develop a structural-acoustics system necessary for further studies in this area. The rest of this paper is organized as follows; in Section 2 the theories behind the developed model and fundamental physics of the system are presented. This is followed by the derivation of the governing equations of the plate, enclosure and coupled system. In Section 3, the modal properties of the plate, enclosure and coupled vibro-acoustic system are simulated using COMSOL software. Finally comparisons between finite element model with the analytical and experimental results obtain in [3] are presented.

\section{Modelling of Sound in an Enclosure}

Producing a sound propagation pattern in an enclosure due to multiple reflections is quite involved. In fact, the response of an acoustic-structure system in the fully coupled case combining the acoustics and structures are quite different from the response of the uncoupled case $[3,4]$. Therefore, in modelling the sound in closed spaces, modal analysis of the enclosure must be performed. In this section, the natural frequencies of the plate and the enclosure in the case of an uncouple condition are examined initially followed by the case of coupled or clamped condition.

2.1. Enclosure with Rigid Walls. Figure 1(a) shows an enclosure with one of its edges located at the origin $0_{c}$ of a Cartesian coordinate system. Here $L_{x c}, L_{y c}$ and $L_{z c}$ are the length, width and height respectively.

The Helmholtz equation, which describes a harmonic wave equation propagating in medium while neglecting dissipation, is represented as

$$
\nabla^{2}+k^{2} p=0
$$

where $p$ is complex sound pressure amplitude and $k$ is the wave number which is related to angular frequency $\omega$ and speed of sound $c_{s}$ by

$$
k=\frac{\omega}{c_{s}} .
$$

Substituting (2) into (1) while introducing air density $\rho_{0}$ [17] gives a homogeneous Helmholtz equation:

$$
\nabla \cdot\left(-\frac{1}{\rho_{0}}(\nabla \mathrm{p})\right)-\frac{\omega^{2} p}{\rho_{0} c_{s}^{2}}=0 .
$$

In addition, the eigenvalue $\lambda$ is related to the eigenfrequency by [17]

$$
\lambda=i 2 \pi f=i \omega .
$$

Substituting (4) into (3) and extending to three dimensions the enclosure can be written as

$$
\frac{\partial^{2} p}{\partial x^{2}}+\frac{\partial^{2} p}{\partial y^{2}}+\frac{\partial^{2} p}{\partial z^{2}}+\frac{\lambda^{2} p}{\rho_{0} c_{s}^{2}}=0 .
$$

The natural frequencies of the acoustical system are obtained by assuming that the boundaries of the enclosure 


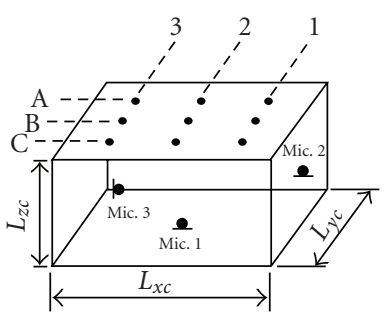

(a)

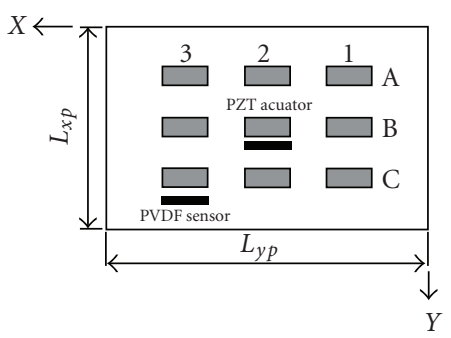

(b)

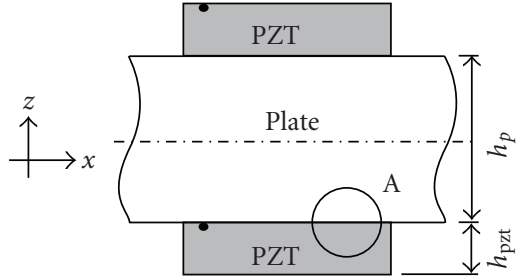

(c)

Figure 2: Experimental model setting and arrangement of piezoelectric, (a) 3D view of enclosure system with microphones, (b) 2D schematic of plate with piezoelectric (c) zone view of symmetric piezoelectric on the plate.

are hard, hence the pressure gradients on all boundaries are set to zero:

$$
\begin{aligned}
& \left.p_{x}\right|_{x=0},\left.\quad p_{x}\right|_{x=L_{x}},\left.\quad p_{y}\right|_{y=0}, \\
& \left.p_{y}\right|_{y=L_{y}},\left.\quad p_{z}\right|_{z=0},\left.\quad p_{z}\right|_{z=L_{z}} \text {. }
\end{aligned}
$$

The solution of (3) with boundary conditions introduced in (6) is given as [2]

$$
\begin{array}{r}
p_{n_{x} n_{y} n_{z}}=A \cos \left(\frac{n_{x} \pi x}{L_{x}}\right) \cos \left(\frac{n_{y} \pi y}{L_{y}}\right) \cos \left(\frac{n_{z} \pi z}{L_{z}}\right), \\
\text { with } n_{x}, n_{y}, n_{z}=0,1,2, \ldots,
\end{array}
$$

where $n_{x}, n_{y}$ and $n_{z}$ are the modes number. Using the derivation in [2], the eigenfrequencies of the enclosure can be further written as

$$
f_{n_{x} n_{y} n_{z}}=\frac{c_{s}}{2}\left[\left(\frac{n_{x}}{L_{x}}\right)^{2}+\left(\frac{n_{y}}{L_{y}}\right)^{2}+\left(\frac{n_{z}}{L_{z}}\right)^{2}\right]^{1 / 2} .
$$

2.2. Flexible Plate. Figure $1(\mathrm{~b})$ shows the position of the flexible plate with a constant thickness $h$. The free harmonic vibration partial differential equation of plate, [18] can be written as

$$
D \nabla^{4} W-\omega^{2} \rho h=0,
$$

where $W(x, y), \nabla^{4}$ and $D$ are the displacement of flexible plate in $x$ and $y$ direction, biharmonic differential operator and bending rigidity respectively. The bending rigidity [18] is given by

$$
D=\frac{E h^{3}}{12\left(1-v^{2}\right)},
$$

where $E, v, \omega$, and $\rho$ are the young's modulus, Poisson's ratio, natural frequency and mass density respectively. The nth vibration mode of the rectangular plate in (9) can be written in a compact form as [18]

$W_{n}(x, y)$

$=\sum_{m=-\infty}^{+\infty}\left[A_{n} J_{n-m}(k x) J_{m}(k y)+B_{n} I_{n-m}(k x) I_{m}(k y)\right]_{\sin }^{\cos }\left(\frac{m \pi}{2}\right)$.
Here, $A_{n}$ and $B_{n}$ are used to satisfy the orthogonality conditions and could be determined by using orthogonal characteristics of these vibration modes [18]. In (11), $J_{n}$ and $I_{n}$ are the Bessel function [18] and the wave number is computed from $k^{4}=\omega^{2} \rho / D$. The expressions (cos) and ( $\left.\sin \right)$ at the right hand side of (11) mean replacing either one of them in this equation appropriately.

Equation (11) is the general solution for the vibration modes of the solid rectangular plate. Here, $J_{n-m}(k x) J_{m}(k y)$ and $I_{n-m}(k x) I_{m}(k y)$ will be used to construct the free vibration solution of a rectangular thin plate with different edge conditions [18]. In this work, fully clamped boundary conditions is assumed and the edge lengths are defined as $a$ and $b$. Therefore, the boundary conditions were defined as

$$
\begin{array}{ll}
\left.W\right|_{x=0}=0, & \left.W\right|_{x=a}=0, \\
\left.W\right|_{y=0}=0, & \left.W\right|_{y=a}=0, \\
\left.\frac{\partial W}{\partial x}\right|_{x=0}=0, & \left.\frac{\partial W}{\partial x}\right|_{x=a}=0, \\
\left.\frac{\partial W}{\partial y}\right|_{y=0}=0, & \left.\frac{\partial W}{\partial y}\right|_{y=a}=0 .
\end{array}
$$

Solving (9) with the clamped boundary conditions yield the vibration modes [18]:

$$
\begin{aligned}
& W_{(n, m)}(x, y) \\
& =\left(A_{n}\left\{J_{n-m}(k x)+J_{n-m}[k(a-x)]\right\}\left\{J_{m}(k y)+J_{m}[k(b-y)]\right\}\right. \\
& \left.\quad+B_{n}\left\{I_{n-m}(k x)+I_{n-m}[k(a-x)]\right\}\right) \\
& \quad \times\left\{I_{m}(k y)+I_{m}[k(b-y)]\right\} \cos \frac{m \pi}{2} \cos \frac{n \pi}{2},
\end{aligned}
$$

where $m$ and $n$ are even numbers.

2.3. Model of the Coupled System. In the case of a coupled system, the effect of the flexible plate on the sound field inside the enclosure as well as the effect of sound field on the flexible plate must be considered together. In coupling the flexible plate to the enclosure, acceleration of the plate was considered as a source of sound. The pressures at rigid boundaries are zero, but at the top of the enclosure where 


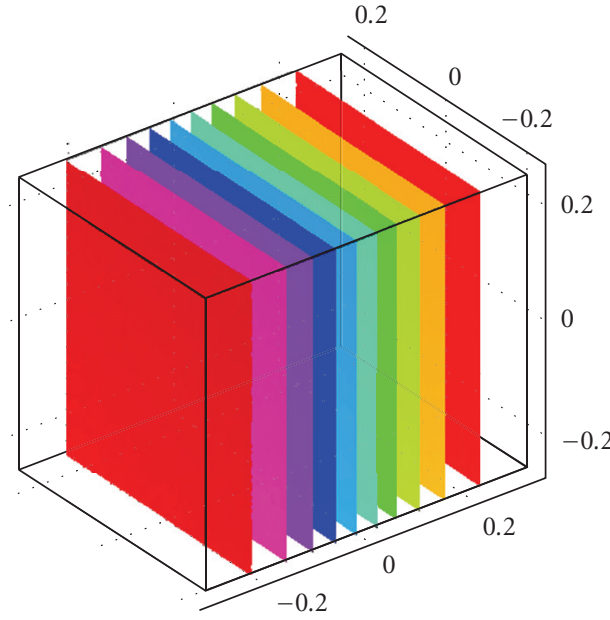

(a)

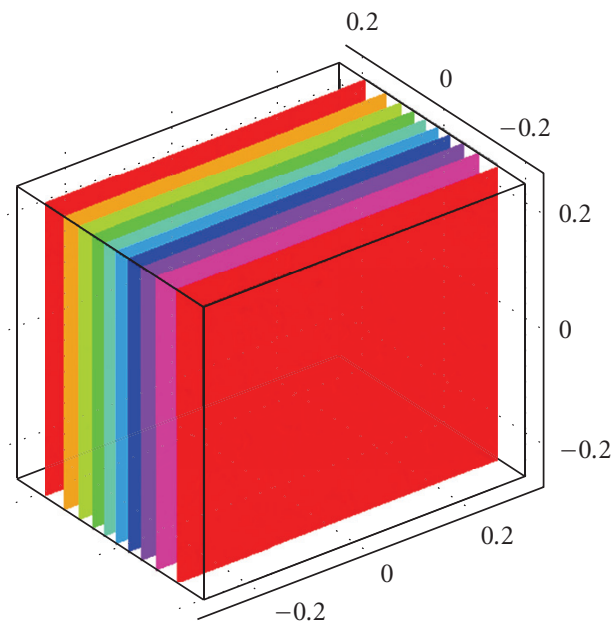

(c)

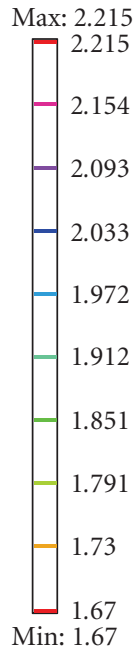

Max: 2.215

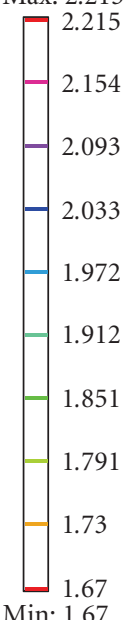

Min: 1.67

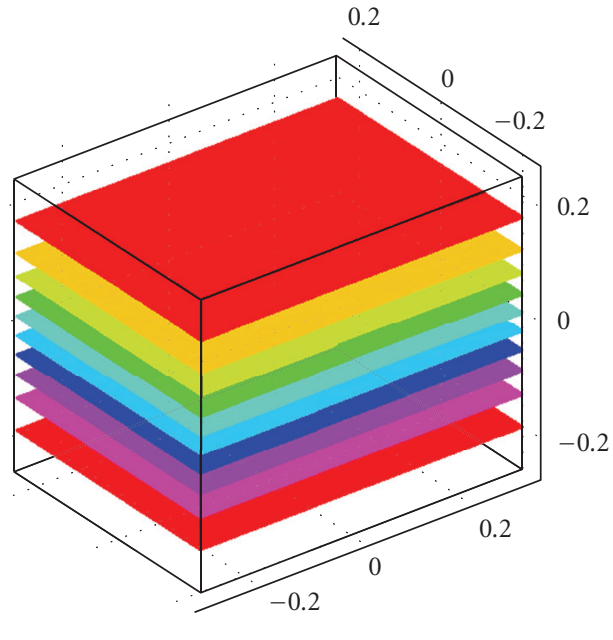

(b)

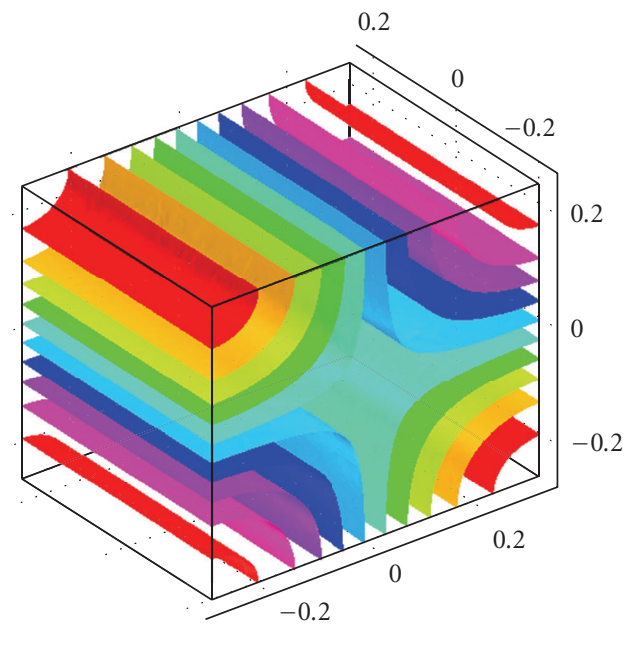

(d)

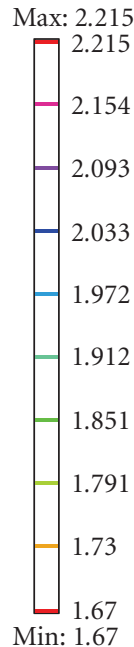

Max: 2.215
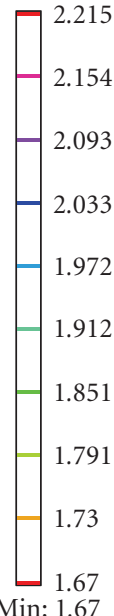

Min: 1.67

Figure 3: A few mode shapes of the rectangular enclosure (a) mode number $(1,0,0)$ at $281.12 \mathrm{~Hz}$, (b) mode number $(0,0,1)$ at $338.98 \mathrm{~Hz}$, (c) mode number $(0,1,0)$ at $379.64 \mathrm{~Hz}$, (d) mode number $(1,0,1)$ at $446.27 \mathrm{~Hz}$.

the flexible plate was placed, it is equal to the acceleration of the plate. Therefore, the homogenous Helmholtz equation (3) becomes [19]

$$
\frac{1}{\rho_{0}}(\nabla \mathrm{p}) \cdot n_{a}=a_{n}
$$

where, $n_{a}$ is the outward-pointing unit normal vector seen from inside the acoustics domain, and $a_{n}$ is the normal acceleration of the plate. Acoustic pressure was also coupled to the flexible plate as a boundary load pressure in the direction of the normal vectors.

As previously mentioned, the combined modal analysis of the coupled system represents the sound propagation of the system with different excitation frequencies. In this work, sound wave is the source of pressure on the plate. The sound waves were generated by a point source. The governing equation for a point source with the power $\mathbf{P}$, and located at the point $\mathbf{R}=\mathbf{R}_{0}$ in an infinite homogeneous space is

$$
\nabla \cdot\left(-\frac{1}{\rho_{0}}(\nabla \mathrm{p})\right)=2 \sqrt{\frac{\pi \mathbf{P} c_{s}}{\rho_{0}} \delta^{(3)}\left(\mathbf{R}-\mathbf{R}_{0}\right)},
$$

where $\delta^{(3)}(\mathbf{R})$ is the Dirac delta function in three dimensional space [19].

\section{Experimental Work in Related Study}

In the experimental studies reported in [3], a rectangular cube with five rigid acrylic sheets was constructed and an aluminium panel was clamped on of it. The Thickness of acrylic sheets and aluminium sheets used were $25.4 \mathrm{~mm}$ and $1.588 \mathrm{~mm}$, respectively. The rectangular cube with the dimension of $L_{x c}=0.6096[\mathrm{~m}], L_{y c}=0.4572[\mathrm{~m}], L_{z c}=$ $0.508[\mathrm{~m}]$ are shown in Figure 2(a), and the plate has the 


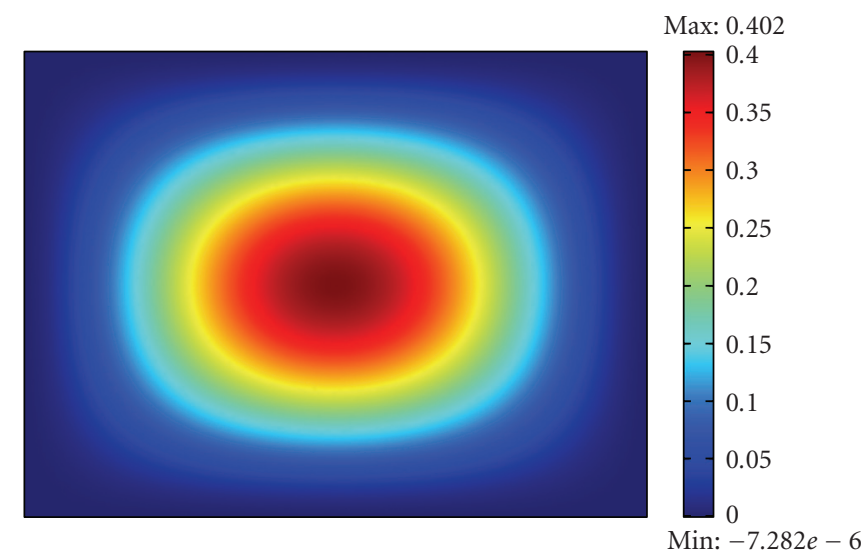

(a)

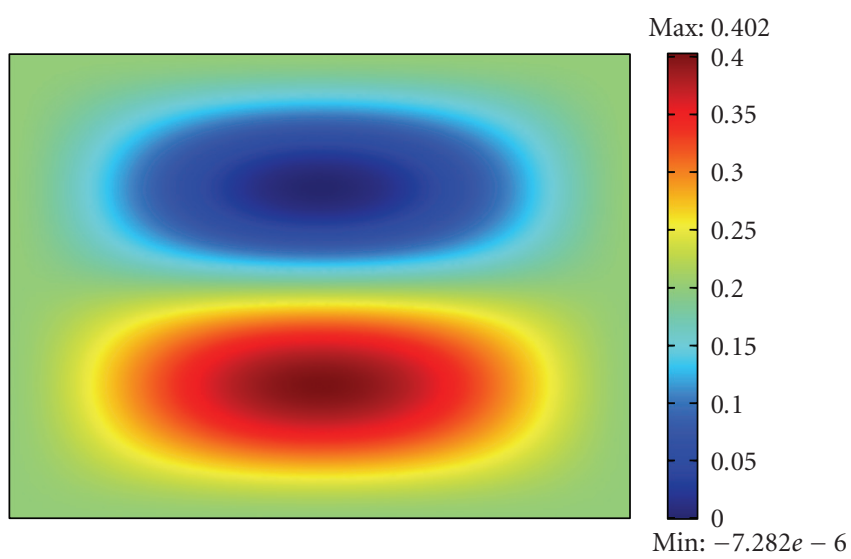

(c)

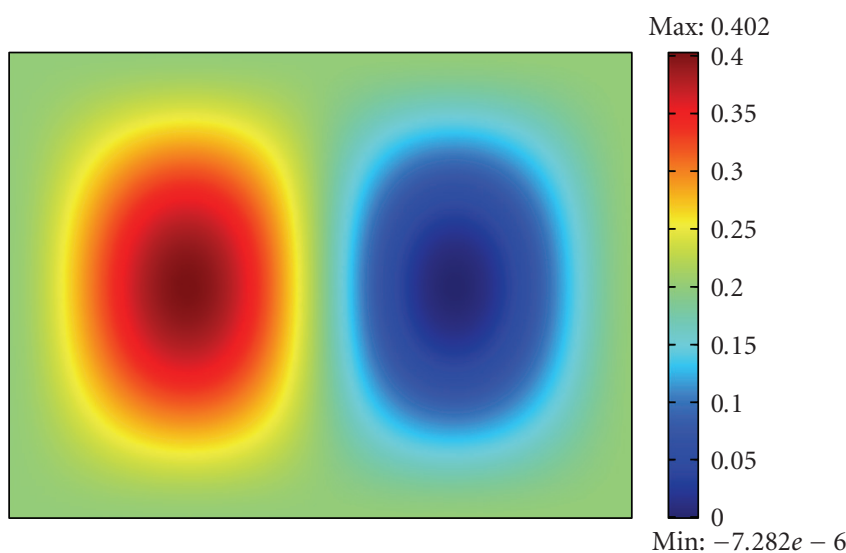

(b)

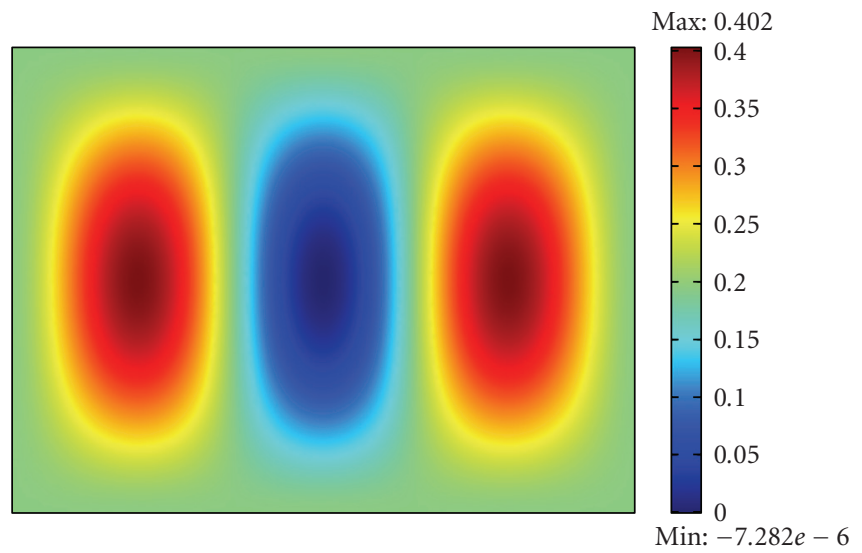

(d)

Figure 4: A few mode shapes of the rectangular plate (a) mode number $(1,1)$ at $44.28 \mathrm{~Hz}$, (b) mode number $(1,2)$ at $75.66 \mathrm{~Hz},(\mathrm{c})$ mode number $(2,1)$ at $103.19 \mathrm{~Hz},(\mathrm{~d})$ mode number $(2,2)$ at $127.05 \mathrm{~Hz}$.

following dimension: $L_{x p}=660.4, L_{y p}=508.0$ which is illustrated in Figure 2(b).

The enclosure was excited by airborne pressure generated by a loudspeaker placed at some distance above of enclosure, and three microphones inside the enclosure were to measure the pressure level inside the enclosure. The location of the microphones inside the enclosure is shown in Figure 2(b). These microphones are used to sense all modes of enclosure using personal computer and dSPACE interface. Nine piezoelectric (PZT-5H) elements were patched symmetrically onto the plate depicted in Figure 2(c).These actuators are used to measure the plate response.

\section{Modelling with COMSOL Multiphysics}

In this section, sound travel into the medium and its interaction with a solid medium are modelled using a finite element software COMSOL Multiphysics. This modelling procedure requires two modules: one for simulating the acoustic medium and the other for flexible plate. After proper selection of the modules, the three dimensional model with the same dimensions described in Section 3 was sketched.
Each module provides a wide range of equations, which was needed in specifying subdomains, boundaries, edges and points. The theories and equations behind this model are based on the governing equations in Section 2.

4.1. Modelling the Enclosure. The "acoustic" module of COMSOL was used to model the enclosure, which utilised partial differential equations based on time harmonic and frequency domain analysis. The boundaries and properties of the medium were set to be hard sound boundary and air characteristics respectively. The dimensions were specified according to the work of [3], which is repeated here:

$$
\begin{gathered}
L_{x c}=0.6096[\mathrm{~m}], \quad L_{y c}=0.4572[\mathrm{~m}], \\
L_{z c}=0.508[\mathrm{~m}] .
\end{gathered}
$$

The point of origin of the enclosure was placed at $(-0.3048,-0.2286,-0.254)$. Using eigenfrequency analysis of the model, the first 4 eigenfrequencies with modal shapes are shown in Figure 3. Due to the hard boundaries used in this analysis, the material of the body of enclosure can be neglected. The shapes of mesh elements were selected 


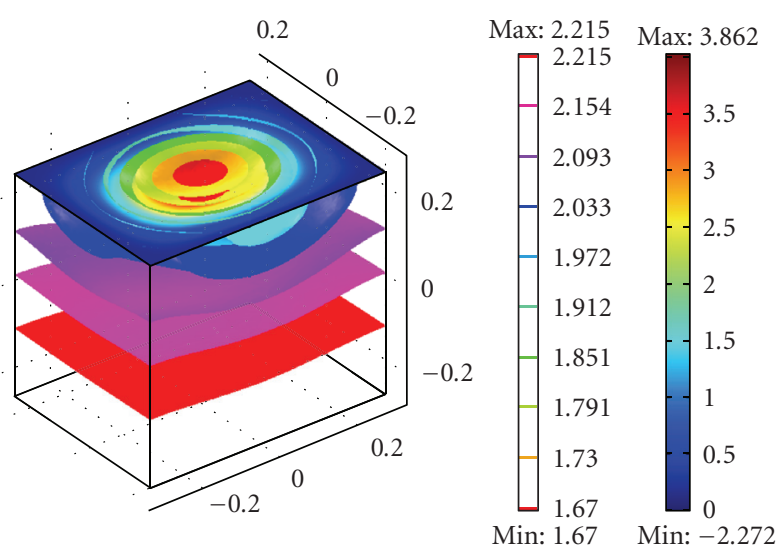

(a)

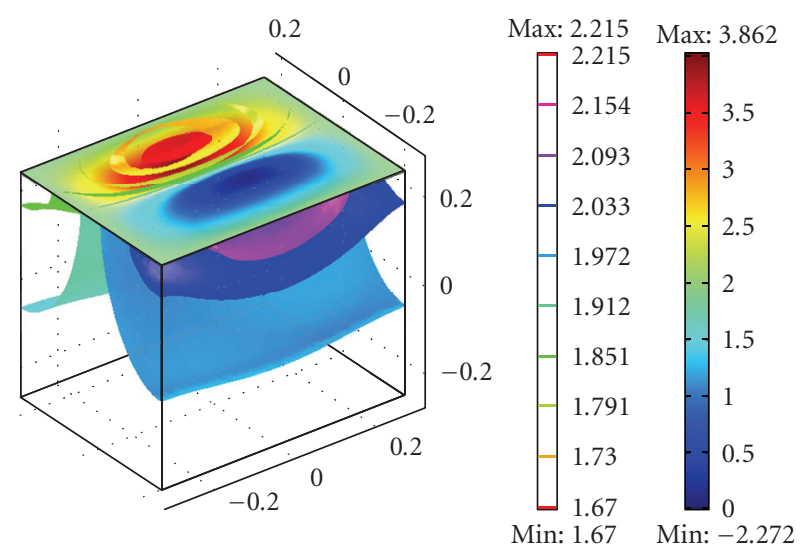

(c)
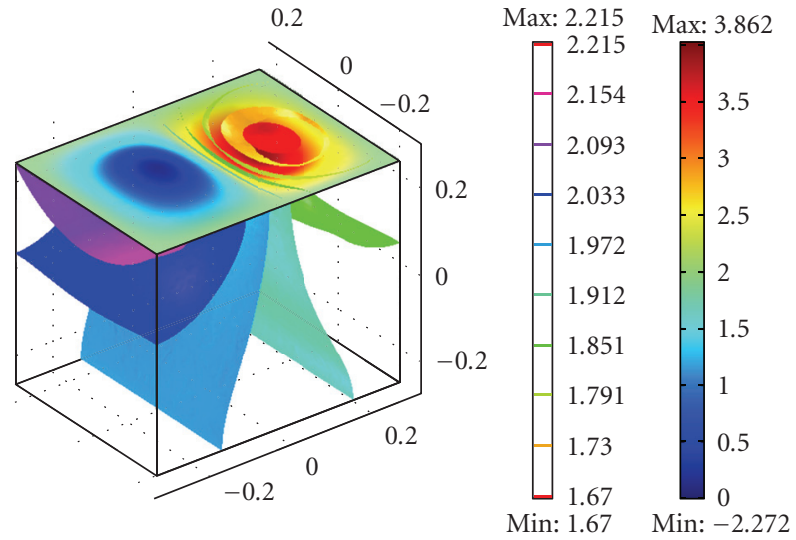

(b)
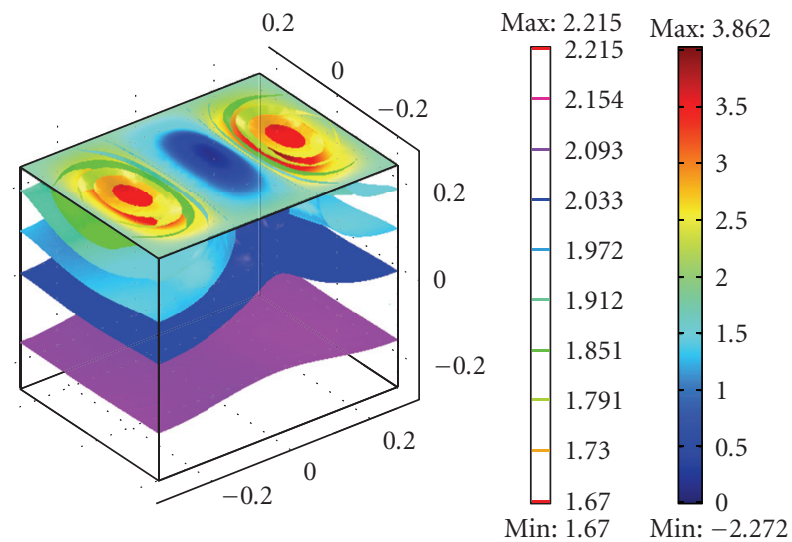

(d)

Figure 5: A few mode shapes of the coupled system (a) first mode at $59.8042 \mathrm{~Hz}$, (b) second mode at $88.878 \mathrm{~Hz}$, (c) third mode at $125.061 \mathrm{~Hz}$, (d) fourth mode at $149.398 \mathrm{~Hz}$.

to be tetrahedral in normal size. The enclosure model consists of 10727 mesh elements. Using the eigenfrequency analysis of the model yield the modal shapes with the first four eingenfrequencies depicted in Figure 3. Solution time and degree of freedom are 24.281 seconds and 56725, respectively.

4.2. Modelling of the Plate. The "Structural Mechanics" module was used to perform the modal analysis of the plate. By adjusting the parameters of the equations, the mode shapes of the solid stress and strain of the plate is plotted in Figure 4. The dimension of the plate is larger than the enclosure, and this is the same as the experimental work of [3]. For this dimension, the coupled mode was constructed as discussed in Section 2.3 .

All edges are selected to be fixed with clamped boundary condition. The shapes of the mesh elements are second order triangular with 18730 mesh elements. Eigenfrequency solver and analysis are selected to solve the model to give the first 17 eigenmodes around $100 \mathrm{~Hz}$. According to this solver, time for solving the model is 17.156 seconds and the number of degree of freedom is 16010. By replacing the
TABle 1: Comparison eigenfrequencies between finite element model with analytical results in [3] for uncoupled system.

\begin{tabular}{lccccc}
\hline \multirow{2}{*}{ Mode } & \multicolumn{2}{c}{ Analytical } & \multicolumn{2}{c}{ Finite element (COMSOL) } & \multirow{2}{*}{ Error \% } \\
& Panel & Enclosure & Panel & Enclosure & \\
\hline$(1,1)$ & 41.6 & - & 44.28 & - & 6.0524 \\
$(1,2)$ & 73.7 & - & 75.66 & - & 2.5905 \\
$(2,1)$ & 95.0 & - & 103.19 & - & 7.9368 \\
$(2,2)$ & 124.6 & - & 127.05 & - & 1.9284 \\
$(1,0,0)$ & - & 281.3 & - & 281.12 & 0.0640 \\
$(0,0,1)$ & - & 337.6 & - & 338.98 & 0.4071 \\
$(0,1,0)$ & - & 375.1 & - & 379.64 & 1.1959 \\
$(1,0,1)$ & - & 439.5 & - & 446.27 & 1.5170 \\
$(1,1,0)$ & - & 468.9 & - & 479.60 & 2.2310 \\
$(1,0,0)$ & - & 504.7 & - & 511.83 & 1.3930 \\
$(1,1,1)$ & - & 577.8 & - & 578.96 & 0.2004 \\
\hline \multicolumn{7}{c}{ Error \% } \\
\hline
\end{tabular}

parameters mentioned above for subdomains and edges, the model is solved and the mode shapes of the plate are shown in Figure 4. 
TABLE 2: Comparison eigenfrequency analysis between simulations, analytical and experimental results.

\begin{tabular}{|c|c|c|c|c|c|}
\hline mode & $\begin{array}{c}\text { Finite element } \\
\text { modelling (FEM) } \\
\text { using COMSOL } \\
\text { software }\end{array}$ & Analytical & Experiment & $\begin{array}{c}\text { FEM } \\
\text { error } 1 \% \\
\text { versus } \\
\text { analytical }\end{array}$ & $\begin{array}{c}\text { FEM } \\
\text { error } 2 \% \\
\text { versus } \\
\text { experimenta }\end{array}$ \\
\hline 1 & 55.11 & 40.9 & 50.1 & 25.7848 & 9.0909 \\
\hline 2 & 82.74 & 72.4 & 74 & 12.4970 & 10.5632 \\
\hline 3 & 116.2 & 93.3 & 91.5 & 19.7074 & 21.2565 \\
\hline 4 & 138.03 & 123.1 & 119.5 & 10.8165 & 13.4246 \\
\hline 5 & 147.01 & 124.1 & 124.5 & 15.5840 & 15.3119 \\
\hline 6 & 196.81 & 174.7 & 172 & 11.2342 & 12.6061 \\
\hline 7 & 216.39 & 194.4 & 192 & 10.1622 & 11.2713 \\
\hline 8 & 227.59 & 203.3 & 194.05 & 10.6727 & 14.7370 \\
\hline 9 & 243.87 & 241.8 & 235 & 0.8488 & 3.6372 \\
\hline 10 & 283.28 & 251.2 & 245 & 11.3245 & 13.5131 \\
\hline 11 & 289.25 & 283.3 & 275.5 & 2.0570 & 4.7537 \\
\hline 12 & 293.49 & 319.5 & 313 & 8.8623 & 6.6476 \\
\hline 13 & 323.72 & 342.3 & 338 & 5.7395 & 4.4112 \\
\hline 14 & 342.03 & 359 & 352 & 4.9616 & 2.9149 \\
\hline 15 & 370.58 & 380.5 & 374 & 2.6769 & 0.9229 \\
\hline 16 & 382.35 & 405.1 & 395.5 & 5.9500 & 3.4393 \\
\hline Average of errors $\%$ & & 一 & & 9.929963 & 9.281338 \\
\hline
\end{tabular}

4.3. Simulation of the Coupled System. The complete coupled system has been simulated and is presented in this section. Two modules (Acoustic and Structural Mechanics) are used in this simulation. The pressure wave source was represented by a point source outside the cavity. A sphere with a reasonably large diameter outside the enclosure was used to envelope the air-filled acoustic domain. On the outer spherical perimeter of the air domain, radiation condition with the spherical wave was used. This boundary condition allows a spherical wave to travel out of the system, giving only minimal reflections for the non-spherical components of the wave. The radiation boundary condition is useful when the surroundings are only a continuation of the domain [19]. Two sub modules from the acoustic tab was used to support thickness and inner dimension of cavity, namely the "Solid stress strain" for thickness and "Pressure Acoustic" for the air inside it. The coupling of flexible plate vibration with the acoustic pressure inside the enclosure and vice versa was performed using both "Acoustic" and "Structure Mechanic" modules. For this purpose, some variables were set to make the connection between these two modules. COMSOL software in such an analysis will enable the combination of different acoustical and vibration phenomena in one model. The variation of some of the mode shapes are depicted in Figure 5.

\section{Results and Discussion}

5.1. Model of the Uncoupled System. The natural frequencies of the system without any coupling between the flexible plate and enclosure were computed from the FEM model. These results are shown in Table 1 and they are compared with those extracted by the analytical model in [3]. The first 11 modes of the system consists of the first four modes of the plate and the first seven enclosure modes. The mean errors of $2.3 \%$ indicate that the results are in good agreement with the analytical model. It is possible to improve the accuracy of the FEM model by decreasing the element size of the mesh applied in finite element modelling. On the other hand, the memory required for this purpose will put a bound on the obtainable accuracy of the model. These limitations are more obvious when comparing the results of couple condition, which requires more element sizes calculations described in the next section.

5.2. Model of the Coupled System. The next step is to calculate the resonant frequencies of the coupled system. The results were compared with both the analytical and experimental results reported in [3]. Table 2 shows the comparison of the coupled system's resonant frequencies between the analytical and experimental model and those obtained using the FEM model. In experimental work rather than rectangular cube and flexible plate, piezoelectric elements are patched to the plate to actuate and control the plate for future applications. The mean of the absolute relative error percentage of 9.929963 is achieved due to comparison with analytical work and 9.281338 percent due to experimental work. The results indicate that finite element method with COMSOL software is able to predict the natural frequency of the whole modes, 
such that active noise control system can be designed using this model. Results that obtained by smaller mesh element sizes gave better results.

In order to improve the accuracy of the model, the mesh element size must be smaller than 0.002 of wavelength (L), where

$$
L=\frac{c_{s}}{f}
$$

This would satisfy the rule of ten to twelve degree of freedom per wavelength. However, this cannot be achieved with our current computing facility (CPU Intel Pentium Dual, 1.60 GHz, Ram, and 2.93 GB). Better computing facility shall be employed in future work.

Note that the analytical results of eigenfrequency analysis of plate and couple condition are taken from Al-bassiouni's method [3].

\section{Conclusions}

Modelling and analysis of the sound-structure interaction of a rectangular enclosure has been addressed in this work. The enclosure has five rigid walls with one aluminium plate as a flexible wall. The effect of a point source noise placed at a distance outside the enclosure on the interior of the enclosure was investigated. The incident noise induced vibrations of the plate, and this in turn generated fluctuation of acoustic pressure inside the enclosure. The modal analysis of the plate, enclosure, and the coupling effects between them were performed and the behaviour of the sound field inside the enclosure was inferred from the shapes of modes at different frequencies for the coupled system. The eigenfrequency analysis showed that the results obtained from the finite element model was reliable with 90\% accuracy when compared with the analytical and experimental results published in related. Therefore, the finite element model can be used further in the studies of active noise control.

\section{List of Symbols}

$L_{x c}, L_{y c}, L_{z c}$ : Dimensions of the enclosure

$p: \quad$ Complex sound pressure amplitude

$k$ : Wave number

$\omega: \quad$ Angular frequency

$c_{s}: \quad$ Speed of sound

$\lambda: \quad$ Eigenvalue

$\rho_{0}: \quad$ Density of air

$n_{a}$ : Outward-pointing unit normal vector

$n_{x}, n_{y}, n_{z}: \quad$ Modes number

$D: \quad$ Bending rigidity

$W(x, y): \quad$ Displacement of flexible plate in $\mathrm{x}$ and $\mathrm{y}$ direction

$\begin{array}{ll}v: & \text { Poisson's ratio } \\ E: & \text { Young's modulus } \\ \rho: & \text { Mass density } \\ I_{n}, J_{n}: & \text { Bessel function } \\ A_{n}, B_{n}: & \text { Constant. }\end{array}$

\section{References}

[1] A. Montazeri, J. Poshtan, and M. H. Kahaei, "Modal analysis for global control of broadband noise in a rectangular enclosure," Journal of Low Frequency Noise Vibration and Active Control, vol. 26, no. 2, pp. 91-104, 2007.

[2] A. Montazeri, J. Poshtan, and M. H. Kahaei, "Analysis of the global reduction of broad band noise in a telephone kiosk using a MIMO modal ANC system," International Journal of Engineering Science, vol. 45, pp. 679-697, 2007.

[3] M. Al-Bassyiouni and B. Balachandran, "Sound transmission through a flexible panel into an enclosure: structural-acoustics model," Journal of Sound and Vibration, vol. 284, no. 1-2, pp. 467-486, 2005.

[4] B. Fang, A. G. Kelkar, S. M. Joshi, and H. R. Pota, "Modelling, system identification, and control of acoustic-structure dynamics in 3-D enclosures," Control Engineering Practice, vol. 12, no. 8, pp. 989-1004, 2004.

[5] M. C. Junger and D. Feit, Sound, Structures and Their Interaction, MIT Press, Cambridge, Mass, USA, 2nd edition, 1986.

[6] E. H. Dowell and H. M. Voss, "The effect of a cavity on panel vibration," AIAA Journal, vol. 1, pp. 476-477, 1963.

[7] E. H. Dowell, G. F. Gorman III, and D. A. Smith, "Acoustoelasticity: general theory, acoustic natural modes and forced response to sinusoidal excitation, including comparisons with experiment," Journal of Sound and Vibration, vol. 52, no. 4, pp. 519-542, 1977.

[8] L. Peretti and E. Dowell, "Asymptotic modal analysis of a rectangular acoustic cavity excited by wall vibration," AIAA Journal, vol. 30, no. 5, pp. 1191-1198, 1992.

[9] B. Balachandran, A. Sampath, and J. Park, "Active control of interior noise in a three-dimensional enclosure," Smart Materials and Structures, vol. 5, no. 1, pp. 89-97, 1996.

[10] H. C. Geng, Z. S. Rao, and Z. S. Han, "New modeling method and mechanism analyses for active control of interior noise in an irregular enclosure using piezoelectric actuators," Journal of the Acoustical Society of America, vol. 113, no. 3, pp. 14391447, 2003.

[11] J. Ro and A. Baz, "Control of sound radiation from a plate into an acoustic cavity using active constrained layer damping," Smart Materials and Structures, vol. 8, no. 3, pp. 292-300, 1999.

[12] D. J. Nefske, J. A. Wolf Jr., and L. J. Howell, "Structuralacoustic finite element analysis of the automobile passenger compartment: a review of current practice," Journal of Sound and Vibration, vol. 80, no. 2, pp. 247-266, 1982.

[13] Y. Y. Li and L. Cheng, "Vibro-acoustic analysis of a rectangular-like cavity with a tilted wall," Applied Acoustics, vol. 68, no. 7, pp. 739-751, 2007.

[14] T. Ran Lin and J. Pan, "Sound radiation characteristics of a box-type structure," Journal of Sound and Vibration, vol. 325, no. 4-5, pp. 835-851, 2009.

[15] C. Q. Howard, C. H. Hansen, and A. Zander, "Vibro-acoustic noise control treatments for payload bays of launch vehicles: discrete to fuzzy solutions," Applied Acoustics, vol. 66, no. 11, pp. 1235-1261, 2005.

[16] A. C. Geerlings, D. J. Thompson, and J. W. Verheij, "Modelbased acoustic substitution source methods for assessing shielding measures applied to trains," Applied Acoustics, vol. 62, no. 8, pp. 979-1000, 2001.

[17] M. J. Crocker, Hand Book of Acoustic, Wiley-IEEE, New York, NY, USA, 2008. 
[18] J. H. Wu, A. Q. Liu, and H. L. Chen, "Exact solutions for freevibration analysis of rectangular plates using Bessel functions," Journal of Applied Mechanics, vol. 74, no. 6, pp. 1247-1251, 2007.

[19] “COMOL MULTIPHYSICS, tutorial, ver. 3.4,” 2007. 

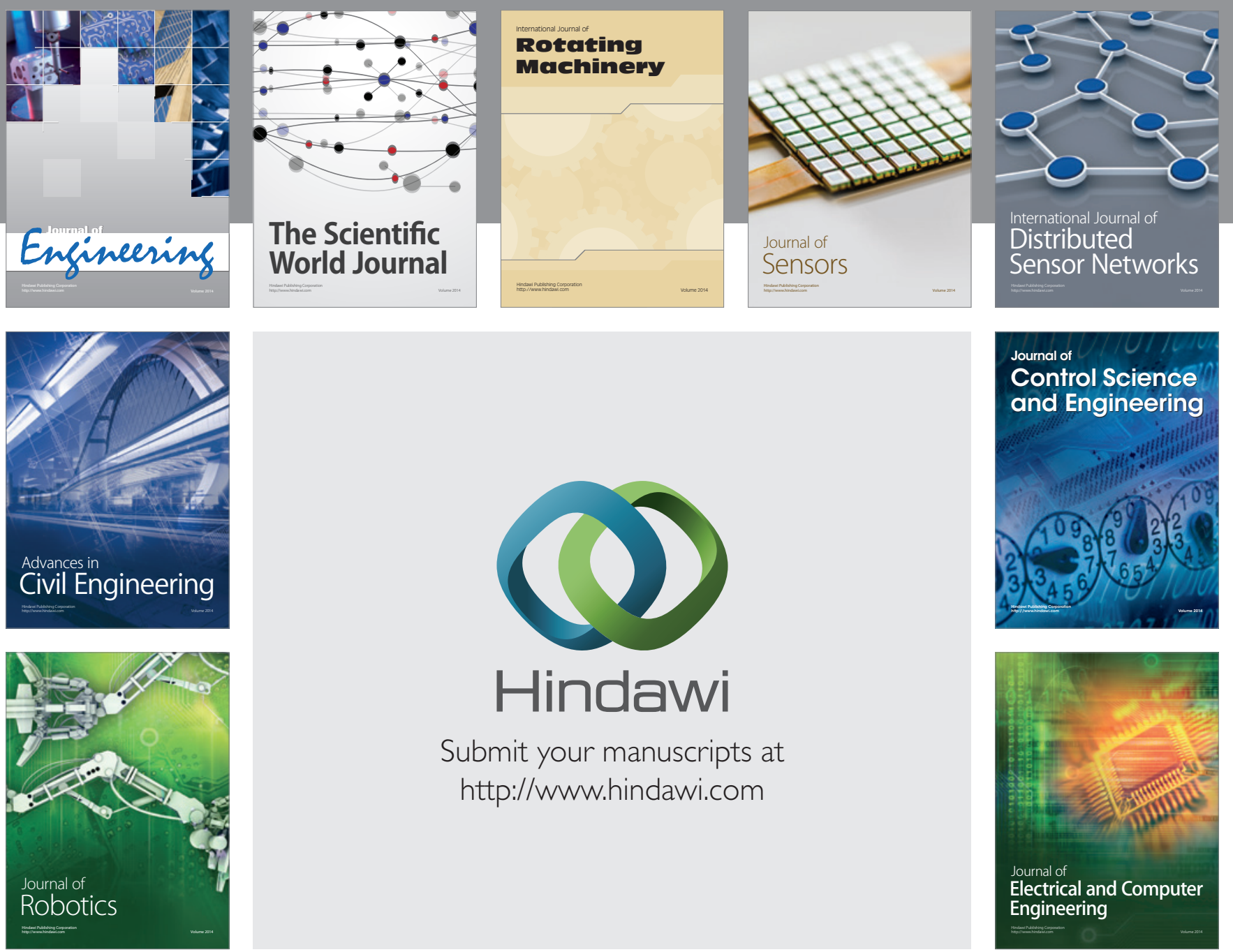

Submit your manuscripts at

http://www.hindawi.com
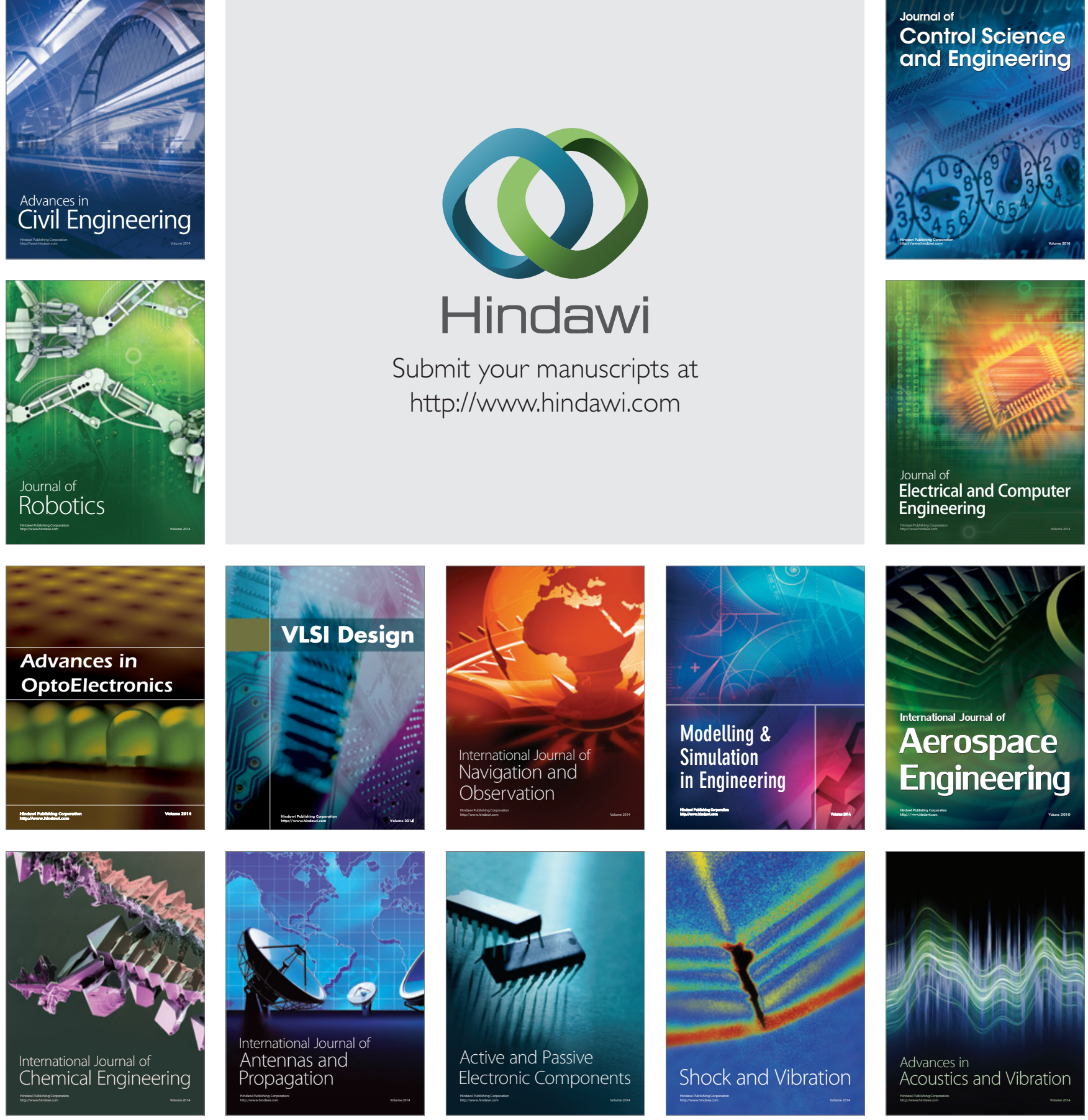